Editorial

\title{
Analyzing Citizen Engagement With European Politics on Social Media
}

\author{
Pieter de Wilde ${ }^{1, *}$, Astrid Rasch ${ }^{2}$, and Michael Bossetta ${ }^{3}$ \\ ${ }^{1}$ Department of Sociology and Political Science, Norwegian University of Science and Technology, Norway \\ 2 Department of Language and Literature, Norwegian University of Science and Technology, Norway \\ ${ }^{3}$ Department of Communication and Media, Lund University, Sweden \\ * Corresponding author (pieter.dewilde@ntnu.no)
}

Submitted: 3 January 2022 | Published: 17 February 2022

\begin{abstract}
Contributions in this thematic issue focus explicitly on citizens and their online engagement with European politics. For social media research in the European Union, citizens remain an understudied actor type in comparison with political elites or news organizations. The reason, we argue, is four key challenges facing social media research in the European Union: legal, ethical, technical, and cultural. To introduce this thematic issue, we outline these four challenges and illustrate how they relate to each contribution. Given that these challenges are unlikely to dissipate, we stress the need for open dialogue about them. A key part of that involves contextualizing research findings within the constraints in which they are produced. Despite these challenges, the contributions showcase that a theoretical and empirical focus on citizens' social media activity can illuminate key insights into vitally important topics for contemporary Europe. These include civic participation, institutional communication, media consumption, gender inequality, and populism.
\end{abstract}

\section{Keywords}

computational methods; European Union; Facebook; gender; news engagement; populism; social media; Twitter

\section{Issue}

This editorial is part of the issue "Analyzing Citizen Engagement With European Politics Through Social Media" edited by Pieter de Wilde (Norwegian University of Science and Technology), Astrid Rasch (Norwegian University of Science and Technology), and Michael Bossetta (Lund University).

(C) 2022 by the author(s); licensee Cogitatio (Lisbon, Portugal). This editorial is licensed under a Creative Commons Attribution 4.0 International License (CC BY).

\section{Introduction}

Within the European Union (EU), the widespread adoption of social media dovetailed with a series of challenges that threatened to undermine the polity. In 2008, a series of economic crises pitted budget contributors against budget receivers, whose citizens rapidly organized anti-austerity protests via social media. In 2014, Eurosceptic politicians skillfully leveraged social media for political campaigning, and their online supporters propelled them to winning over one-fourth of the seats in the European Parliament. Shortly thereafter, tragic scenes of a migrant influx from the Middle East went viral on social media, generating both empathy and anti-Muslim hostility. Today, in an EU comprising one less member state, concerns mount about the role of social media in amplifying conspiracy theories and misinformation in the midst of a public health crisis.

While social media has been integral to the EU's recent trajectory, academic research investigating the relationship between EU citizens and social media has proven difficult to conduct. To date, both citizens as an actor-class and the European dimension of their social media activity have remained under-researched. We asked the academic community to carry out research projects that specifically focus on citizens and their engagement with European politics on social media. The result is an inspiring collection of articles on issues such as populism, gender, online engagement, news consumption, and data accessibility.

In opening this thematic issue, we present our reading of the state of social media research on the EU to situate 
the research on citizens along three dimensions: content, context, and activity. When analyzing social media content, scholars interested in studying European politics have adopted two main prisms for conceptualizing Europe in their research designs: vertical and horizontal. Whereas research adopting a vertical dimension examines the extent to which the content of online discussions explicitly mentions EU-level actors, issues, and processes, the horizontal dimension examines the extent to which citizens' social media engagement within national public spheres is comparable across borders. Social media studies that focus on citizens have, by-and-large, taken the horizontal approach through comparative research designs of national-level phenomena that relate to Europe, rather than a direct analysis of citizens' social media activity as it pertains to the vertical dimension: Brussels, EU institutions, or pan-European events.

Whether scholars approach Europe through a vertical or horizontal lens, the context of their empirical cases can be divided into two groups: formal and extra-parliamentary politics. Understandably, formal electoral research tends to revolve around the European Parliament elections and, following broader trends in political communication, focuses almost exclusively on political elites. Most of the research on European citizens and social media concentrates around extra-parliamentary politics, such as protest mobilization, discursive participation around controversial topics, and patterns of engagement with political news.

In addition to content and context, we can broadly categorize prior research on citizens based on whether they frame citizens' online activity as proactive or reactive. Studies of proactive online engagement examine the role of social media in citizen-initiated content, such as the coordination of protests or starting online debates about issues of transnational relevance. Reactive online engagement, meanwhile, refers to studying citizens' social media activity in response to preexisting content, such as commenting on media articles or reacting to politicians' posts. Studies examining citizens' unprompted, proactive social media conversations find relatively high levels of vertical Europeanization, whereas reactive conversations tend to remain confined to cross-border or domestic issues with little attention to the vertical level.

Synthesizing the results of existing research on social media and European politics in this way, we identify three biases in current approaches to the subject. First, studies on citizens are heavily weighted toward comparative case studies of national contexts, rather than an explicit focus on the EU within these contexts. Second, an actor-type discrepancy exists between studies of formal and extra-parliamentary political processes. Formal electoral studies overly focus on political elites, and therefore our knowledge of citizens' social media activity in the EU is primarily limited to extra-parliamentary politics. Third and related, studies on citizens tend to focus on citizens' proactive engagement with pan-European topics.
To a large extent, these biases can be attributed to four challenges in conducting academic research on citizens' engagement with European politics through social media: legal, ethical, technical, and cultural. We detail these challenges below, and illustrate how each contribution in this thematic issue grappled with them. Thus, our aim with this thematic issue is to contribute both to our substantive knowledge on citizen engagement with European politics as well as our understanding of the current practice of academic research on the theme.

\section{Legal Challenges}

The cottage industry of opinion mining that started in the early 2010s generated increasing push back in the second half of the 2010s. Privacy agents rang alarm bells, critics warned about the consequences for democracy, and regulators sharpened legislation and oversight. Legal barriers were erected that make it more difficult for commercial interests and researchers to access social media data. In particular, the Cambridge Analytica scandal of 2017 highlighted the ethical complications of studying people's political opinions through social media without the informed consent of users.

The General Data Protection Regulation (GDPR) - the main piece of EU legislation governing privacy onlineprovides two routes for accessing what people post on social media. The first route is through informed consent. This requires an academic to inform individuals of the research project conducted and get them to agree to being studied. This is extremely arduous given anonymity on social media, difficulties in contacting individuals, and the sheer amount of people who would need to agree to participate in order to make quantitative analysis of the vast amount of material available possible. The second route is through an appeal to the public interest in understanding the role that social media plays in democracy and political accountability. In practice, a norm has developed that this second route allows for studying the behavior of political elites such as political parties and individual politicians online. But it does not allow for studying citizen engagement directly, in the form of analyzing the comments and posts ordinary citizens leave behind online. This will have to be approached indirectly, for example in an aggregated manner through studying the amount of likes that posts by political elites gather.

The contributors to this thematic issue clearly shied away from citing individual posts by citizens online. Heidenreich et al. (2022, p. 129) explicitly articulate how GDPR limits our ability to study citizen engagement with European politics through social media:

This [GDPR privacy restriction] particularly concerns the actual comments. It is currently impossible to gather information on this aspect, barring any research on the actual contents posted in response to status posts....This also means that we cannot assess what the engagement with EU news, as 
operationalized in this study, means in terms of legitimacy or support for the EU as such.

\section{Ethical Challenges}

Ethical considerations have tended to be an afterthought in social media research. In the early days of social media, the idea that there might even be an ethical component tended to be neglected. Zimmer and Proferes (2014, p. 256) found that only $4 \%$ of published articles on Twitter made any explicit mention of ethical considerations - a third of which only to conclude that Twitter data was "public information and thus its collection and use did not require ethical review or special consideration." While attention to ethics may have increased since then, their findings reveal a research community not intuitively attuned to thinking about ethics. As ethics review boards and various field-specific organizations have developed ethical guidelines for a constantly shifting terrain of social media platforms and practices, it is perhaps the still stricter formal GDPR constraints discussed above that have particularly forced researchers to consider the privacy of the people they are studying. For example, Seibicke and Michailidou (2022, p. 102) state: "We refrained from directly identifying and quoting (eponymously or anonymously) individuals," in order not to breach GDPR privacy rules.

A number of studies have pointed to the discrepancy between researchers' perception of the publicity of the data they work with and users' perceptions of their generated content. As Fiesler and Proferes (2018, p. 2) found by asking social media users directly: "The majority of Twitter users in our study do not realize that researchers make use of tweets, and a majority also believe that researchers should not be able to do so without permission." Clearly, this observation is far removed from many researchers' assumptions that anyone who posts on Twitter must be aware that tweets are public and can be used in research. While it would be easy for scholars to point to the Terms and Conditions of the platform, it is well established that these lengthy and changing documents are rarely read or understood by users (Beninger et al., 2014, p. 14). Ethically, if not legally, researchers are obliged to consider whether users can "reasonably expect to be observed by strangers" (Townsend \& Wallace, 2016, p. 10). Indeed, researchers may even find themselves torn between following the legal requirement set by Twitter in their Terms and Conditions (i.e., to cite the full text and user handle) and ethical considerations about maintaining users' anonymity (Beninger et al., 2014, p. 33).

The need for ethical awareness increases when it comes to the content produced by vulnerable subjects or regarding sensitive topics. Williams et al. (2017) found that groups that are more likely to be exposed to online harassment such as women, LGBT people, and Black or minority ethnic tweeters were more likely to feel concerned about their posts being used for research. A basic requirement in ethical guidelines is that one should avoid exposing people to risk of harm (Townsend \& Wallace, 2016, p. 7). For vulnerable groups, such risk of harm is bound up with their identity so that publicizing the very existence of a person who is Black or gay may put them at risk by potentially bringing them to the attention of trolls or violent groups. Hence, Galpin (2022, p. 168) argues that "the nature of subaltern counter-spheres is often that they cannot be ethically studied without the explicit consent and approval of community members and may need to involve researchers who belong to the communities being analysed."

Regarding ethical challenges, let us finally raise a critical reflection on the relationship between the researcher and the platform. As Srinivasan (2017, p. 1) argues:

We treat commercial platforms such as Facebook, Twitter, or Google today as if they were public spaces and systems, ignoring that they must remain primarily accountable to their shareholders. These commercial priorities, rather than the diverse publics and cultures, shape how these tools are developed and the agendas they serve.

On the one hand, this should prompt researchers to consider the influence of such agendas on the politics that we study. Who gets to say what, given the agendas and algorithms of the platforms? On the other hand, it should make us scrutinize our own position as researchers. To what extent does our dependence on the platforms to get access to data prevent us from asking critical questions about them? While most contributions in this thematic issue treat platforms as a window through which we can study the political behavior of, and interaction between, political elites and citizens in Europe, Dommett and Tromble (2022) explicitly pick up the role of platforms and the fact that they are not "fully transparent windows." They argue for more academic activism to force platforms to provide access and illustrate how this may be done.

\section{Technical Challenges}

The relationship between researcher and platform also highlights some of the key technical challenges in studying social media. Researchers, too, are end-users of social media platforms, albeit in a different way than users interfacing with platforms on the front-end. When it comes to researchers' capacity to study social media, our reliance on platform data has been laid bare, most notably through Facebook's throttling of public access to its Graph API. Yet, scholarly reflections in response to that inflection point have generated a stronger critical awareness about how the data made available by platforms subtly work to steer our objects of analysis. This nudging represents the overarching technical challenge to overcome when studying citizens' engagement on social media. 
While computational techniques to analyze social media data present a technical challenge for researchers, these barriers are within researchers' control and are thus the easiest to overcome. Like with any methodology, computational methods applied via programs like $\mathrm{R}$ or Python require training, practice, and learning-by-doing. Instead, we focus here on more fundamental technical challenges in social media research that relate, in one way or another, to data access and structure. The scale, format, and availability of this data have direct implications for our understanding of citizens' online political engagement.

The first technical challenge is scale. Platforms structure data access to both academics and citizen users alike, in ways that obscure an aggregate-level understanding of citizens' political engagement on social media. Scale, in terms of processing large datasets, can to some extent be solved with increased computational power. However, even if it is possible to gather vast amounts of data, storing and sharing data amongst international collaborative research teams is not without its obstacles, as privacy regulations generally prohibit the storage of social media data. As noted by Özdemir and Rauh (2022, p. 142):

Managing...large volumes of data entails major logistical problems with regard to storing, sharing, and analyzing the data-especially in a collaborative project. While collaborative coding is tremendously facilitated by services such as our preferred GitHub, such free-of-charge services quickly reach their limits with the amount of data we had to wrangle for the analyses here.

Scale, and how platforms deal with it, is also a challenge as it affects what citizens see on social media. Algorithmic filtering is a widespread business practice to manage and personalize content, resulting in the research limitation noted by Bil-Jaruzelska and Monzer (2022, p.182): "We lack insight into how algorithms promote content and thus influence engagement, which limits our ability to control for confounding factors that drive engagement."

A second technical challenge is the availability of data, where the power of platforms to influence research on citizens is most visible. On the one hand, platforms can entirely limit access to citizens' data in line with legal and ethical considerations outlined above. For example, Facebook has historically blocked data access to citizens' private networks, and more recently has revoked access to citizens' comments on public pages due to privacy concerns. Thiele (2022, p. 193) notes that:

Accessing the Facebook API has become more and more difficult for researchers in the past few years. Many social media scholars today are dependent on endeavors like Facepager....Such programs, however, have a precarious status themselves and constantly run the risk of losing the access granted by
Facebook. A different problem is the lack of transparency and constant changes of the Facebook API. The data returned sometimes exhibit gaps or skewness for unclear reasons.

The unavailability or uncertainty regarding data completeness not only limits our understanding of citizens' political engagement on the platform, it also drives researchers to focus on Twitter. Platforms' current approach to addressing this technical challenge is the public release of official datasets using anonymization techniques such as differential privacy. Researchers interested in citizens' political engagement should, however, consider the implied normative valence of these datasets. To date, publicly released datasets focus predominantly on understanding disinformation, either through understanding the dissemination strategies of malicious actors (Twitter's Influence Operations datasets) or citizens' fake news sharing (Facebook URLs dataset). Especially in the latter case, access to data is conditional upon researching citizens' misinformation sharing, which steers research toward citizens' negative practices of social media engagement. When seeking to understand the democratic implications of citizens' social media engagement, researchers should also consider how social media activity can positively contribute to democratic norms. But this requires linking very abstract concepts like legitimacy or deliberation to the short and abbreviated reality of actual social media posts. The next section picks up these cultural challenges.

\section{Cultural Challenges}

By focusing specifically on European politics, this thematic issue fills one of the important gaps in the research about politics on social media. As Bruns et al. (2016, p. 2) note, the US (and to a lesser extent the UK and other Western countries) are overrepresented in the literature on social media and politics. They point out that the American political and media systems are so particular that it is hard to translate the findings on how media are used for political campaigning there to other contexts. "What is necessary instead," they suggest, "is a broadbased, cross-national investigation of social media use in political communication and campaigning that allows for a charting of the similarities and differences in social media adoption and application against the backdrop of specific national...contexts" (Bruns et al., 2016, p. 2).

This is a challenge that many of our contributions pick up on, for example Bene et al. (2022). Wallaschek et al. (2022) illustrate the problems with such a crossnational approach. Data in small countries might not be enough, such as on International Women's Day 2021 in Lithuania. Spain and Ireland have languages that are spoken in multiple countries, making language-based sampling problematic. In short, "Collecting cross-country social media data remains a challenge" (Wallaschek et al., 2022, p. 157). 
A key difference here is between cross-national and transnational studies, with the former placing nations alongside one another while the latter is interested also in connections at the sub- and supranational levels (Struck et al., 2011). People engage in political activities and conversations on social media in groupings that do not neatly align with the national community, sometimes being closely connected to their local neighborhood. At other times, this engagement cuts across state borders. Approaching politics on social media through a transnational lens allows those connections between citizens at the local, regional, and supranational levels to come into focus. Bossetta et al. (2017, p. 54) refer to the "transnational promise" of social networking sites "to contribute to instantaneous, cross-border flows of political communication." Such transnational dynamics are explicitly picked up by Özdemir and Rauh (2022) in their study of supranational communication by EU institutions, and by Seibicke and Michailidou (2022) in their reflections on how forms of association with the EU are debated in various countries.

Yet, when carrying out research into such transnational phenomena, we are faced with a number of cultural challenges. Broadly speaking, these relate to the feasibility and desirability of transnational and comparative research. To what extent is it feasible (given the language skills and resource limitations of the researcher) to study different European contexts in one project? And to what extent can these places be meaningfully compared, given the differences in both political culture and social media culture, and what are the pitfalls such a project must avoid?

The first challenge of transnational or comparative research is its feasibility. As researchers in fields like transnational history and comparative literature have discovered, to their chagrin, the scope of a project can become too big to be practicable. Just as there are only so many archives one historian can go to, or so many languages one literary scholar can read, so too are there practical limits that constrain the ambitions of the social media researcher. We do our best to select relevant cases and comparisons, but it remains difficult to assess the generalizability of findings outside of the chosen cases (González-González et al., 2022; Hameleers, 2022). While some of these obstacles may be dealt with by recruiting someone with the necessary skills, the very real problem of funding will often mean that the only solution becomes to scale down the ambitions of the project. Large collaborative projects, such as the one by Bene et al. (2022), make major steps in expanding the cultural space under study. Yet, a single study that includes all European countries remains elusive, and limitations in obtaining EU-wide, comparable information on how politics on social media is similar or different across member states mean we have to generalize to the entire European continent with caution.

Furthermore, we must consider possible limits to the desirability of working across borders. Is there a risk that in the effort to paint the bigger transnational picture we lose local nuances? While European citizens may communicate and organize across national borders, they are still affected by the social and political realities that apply locally and which may affect social media use. Attention to the border-crossing potential of social media should not lead us to fall into the trap of what Chan (2013, p. xi) terms "digital universalism," where we assume that digital culture is the same everywhere. While Chan's research focuses on Peru, the warning applies also to a European framework. Since much existing research on European social media usage focuses on Western Europe, there is a risk in treating one country as a generalizable case, and subsequently comparing to what extent the rest of Europe follows or diverges from one country's model. Any transnational study of social media use must be sensitive to local practices and take care not to see them as derivative of a Western standard.

\section{Conclusion}

While we are not able to overcome all four of these research challenges in this thematic issue, our aim is to illustrate how a core focus on European citizens' social media engagement can advance social science across multiple fronts: political studies, media studies, and gender studies. Since the outlined challenges are unlikely to dissipate, we stress the need for open dialogue and knowledge sharing about them, so that the strength and limitations of research findings can be contextualized within the constraints in which they are produced. For social media research, the accumulation of knowledge requires not only theoretical and empirical development; it also requires the development and sharing of best practices to overcome the challenges we outline here. In the spirit of open science, we present these challenges at the outset of this thematic issue, and we invite readers to reflect upon them as they read the contributions herein.

\section{Acknowledgments}

We gratefully acknowledge funding for the project Trondheim Analytica, as part of NTNU's Digital Transformation initiative, that has enabled this thematic issue.

\section{Conflict of Interests}

The authors declare no conflict of interests.

\section{References}

Bene, M., Magin, M., Jackson, D., Lilleker, D., Balaban, D., Baranowski, P., Haßler, J., Kruschinski, S., \& Russman, U. (2022). The polyphonic sounds of Europe: Users' engagement with parties' European-focused Facebook posts. Politics and Governance, 10(1), 108-120. Beninger, K., Fry, A., Jago, N., Lepps, H., Nass, L., \& Sil- 
vester, H. (2014). Research using social media; users' views. NatCen Social Research.

Bil-Jaruzelska, A., \& Monzer, C. (2022). All about feelings? Emotional appeals as drivers of user engagement with Facebook posts. Politics and Governance, 10(1), 172-184.

Bossetta, M., Dutceac Segesten, A., \& Trenz, H.-J. (2017). Engaging with European politics through Twitter and Facebook: Participation beyond the national? In $\mathrm{M}$. Barisione \& A. Michailidou (Eds.), Social media and European politics: Rethinking power and legitimacy in the digital era (pp. 53-76). Palgrave Macmillan.

Bruns, A., Enli, G., Skogerbo, E., Larsson, A. O., \& Christensen, C. (2016). Introduction. In A. Bruns, G. Enli, E. Skogerbo, A. O. Larsson, \& C. Christensen (Eds.), The Routledge companion to social media and politics (pp. 1-3). Routledge.

Chan, A. (2013). Networking peripheries: Technological futures and the myth of digital universalism. MIT Press.

Dommett, K., \& Tromble, R. (2022). Advocating for platform data access: Challenges and opportunities for academics seeking policy change. Politics and Governance, 10(1), 220-229.

Fiesler, C., \& Proferes, N. (2018). "Participant" perceptions of Twitter research ethics. Social Media + Society, 4(1). https://doi.org/10.1177/20563051187 63366

Galpin, C. (2022). At the digital margins? A theoretical examination of social media engagement using intersectional feminism. Politics and Governance, 10(1), 161-171.

González-González, P., Marcos-Marné, H., Llamazares, I., \& Gil de Zúñiga, H. (2022). The informational consequences of populism: Social media news use and "news finds me" perception. Politics and Governance, 10(1), 197-209.

Hameleers, M. (2022). Empowering the people's truth through social media? (De)legitimizing truth claims of populist politicians and citizens. Politics and Governance, 10(1), 210-219.

Heidenreich, T., Eisele, O., Watanabe, K., \& Boomgaarden, H. G. (2022). Exploring engagement with EU news on Facebook: The influence of content characteristics. Politics and Governance, 10(1), 121-132.

Özdemir, S., \& Rauh, C. (2022). A bird's eye view: Supranational EU actors on Twitter. Politics and Governance, 10(1), 133-145.

Seibicke, H., \& Michailidou, A. (2022). The challenges of reconstructing citizen-driven EU contestation in the digital media sphere. Politics and Governance, 10(1), 97-107.

Srinivasan, R. (2017). Whose global village? Rethinking how technology shapes our world. New York University Press.

Struck, B., Ferris, K., \& Revel, J. (2011). Introduction: Space and scale in transnational history. The International History Review, 33(4), 573-584.

Thiele, D. (2022). Pandemic populism? How Covid-19 triggered populist Facebook user comments in Germany and Austria. Politics and Governance, 10(1), 185-196.

Townsend, L., \& Wallace, C. (2016). Social media research: A guide to ethics. University of Aberdeen.

Wallaschek, S., Kaushik, K., Verbalyte, M., Sojka, A., Sorci, G., Trenz, H.-J., \& Eigmüller, M. (2022). Same same but different? Gender politics and (trans-)national value contestation in Europe on Twitter. Politics and Governance, 10(1), 146-160.

Williams, M. L., Burnap, P., \& Sloan, L. (2017). Towards an ethical framework for publishing Twitter data in social research: Taking into account users' views, online context and algorithmic estimation. Sociology, 51(6), 1149-1168.

Zimmer, M., \& Proferes, N. J. (2014). A topology of Twitter research: Disciplines, methods, and ethics. Aslib Journal of Information Management, 66(3), 250-261.

\section{About the Authors}
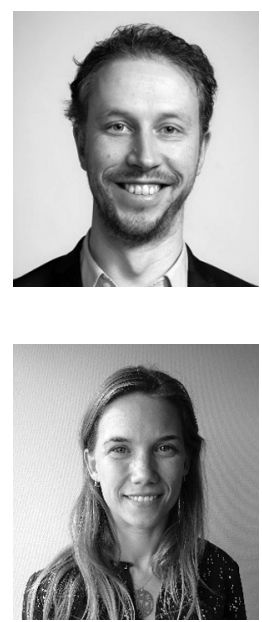

Pieter de Wilde is professor in political science at the Norwegian University of Science and Technology (NTNU), Trondheim, Norway. His research focuses on political conflict over European integration and globalization. This includes the politicization of European integration, EU responsiveness to public opinion, the role of national parliaments in the EU, Euroscepticism, and the extent to which new politics issues are embedded within a broader cleavage. He is principal investigator for the projects "Unelected representatives: The impact on liberal democracy in Europe," "Trondheim Analytica," and "Reconciling Europe with its citizens through democracy and rule of law (RECONNECT)."

Astrid Rasch is associate professor of Anglophone cultural studies at the Department of Language and Literature at the Norwegian University of Science and Technology. Her research examines post-imperial memory politics in Zimbabwe, Australia, the Caribbean, and Britain. She is the co-editor of Embers of Empire in Brexit Britain (with Stuart Ward, Bloomsbury, 2019). She is the principal investigator of "Literatures of change: Culture and politics in Southern Africa" and the interdisciplinary social media project "Trondheim Analytica." 


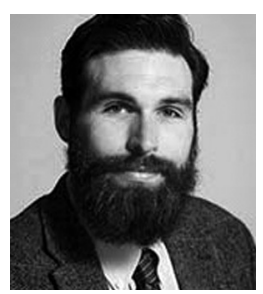

Michael Bossetta is assistant professor in the Department of Communication and Media at Lund University. His research interests largely revolve around the intersection of social media and politics, particularly in terms of political campaigning and participation. He produces and hosts the Social Media and Politics podcast, available on any podcast app. You can follow him on Twitter @MichaelBossetta and the podcast @SMandPPodcast. 